\title{
Jell- $\mathrm{O}^{\circledR}$ microfluidics and synthetic biology: combining science outreach with basic research
}

"With these outreach activities we have engaged students in science who would not otherwise have considered it as a career."

Keywords: education $=$ Jell-O ${ }^{\circledR}=$ microfluidics $\approx$ science outreach $\approx$ synthetic biology

We have found that integrating basic science and engineering research with teaching and outreach initiatives offers rewards to learners and teachers, both in university settings and in industry. We present here two examples of this coordination in action, the undergraduate International Genetically Engineered Machines (iGEM) competition [101] and Jell- ${ }^{\circledR}$ microfluidics [1]. In contrast to recent literature suggesting that the integration of research and teaching within conventional academic paradigms is difficult to impossible $[2,3]$, we have found that such integration is possible, but requires unique settings and approaches different from either traditional research or teaching. Our particular setting, the Michael Smith Laboratories (MSL; Canada), provides one successful model of how this combination can be effectively coordinated. The benefits to the academic and industrial communities include intellectual stimulation, recruitment of excellent students, increased interdisciplinary collaboration and enhanced 'soft skills', critical to any successful career in science, for both teachers and learners. Students' authentic experiences in this approach are tied to real research, the resulting focus on hands-on learning increases student engagement and positive learning outcomes.

The interdisciplinary setting of the MSL has provided us with a unique venue for displaying the value of our research to the public. Named after Michael Smith (1993 Nobel prize winner in chemistry), the MSL houses research laboratories, educational facilities and space open to the public, and is home to research groups from across ten different departments at the University of British Columbia (UBC), Canada. By recruiting engineers and scientists with research programs that cut across disciplines, Michael purposely set out to create this environment where shared resources and collaborations are the norm. This philosophy of sharing was aptly demonstrated by Michael himself when he donated the entire proceeds from his Nobel prize to support schizophrenia research, the advancement of women in science and science outreach. Inspired by Michael, the MSL operates today as a collaborative research environment that values research excellence as well as successful science outreach programming. This provides us with a unique setting for launching creative educational initiatives that highlight the value of interdisciplinary research.

One of the science education projects we have engaged in recently is the formation of an undergraduate synthetic biology team. The team competes in the annual iGEM competition at MIT. iGEM seeks the answer to the question: "Can simple biological systems be built from standard, interchangeable parts and operated in living cells, or is biology simply too complicated to be engineered in this way?" Since the inception of iGEM in 2006, it has grown to 128 teams from 24 countries, and many useful technologies have been introduced, including some published in leading journals $[4,5]$. The MSL serves as an ideal vehicle for iGEM at UBC. The educational facilities at the MSL (including a fully equipped molecular biology teaching laboratory) and the MSL core facilities (warm rooms, cold rooms, incubators and centrifuges) are available for the students' use. In our first year, the UBC team was awarded a gold medal for its work on the design and implementation of an analog threshold biosensor. As well as cultivating research careers for the top science students at UBC, this program has added to the richness of the MSL in two ways. First, it has encouraged and strengthened interactions between faculty members across campus with expertise in synthetic biology. Second, it

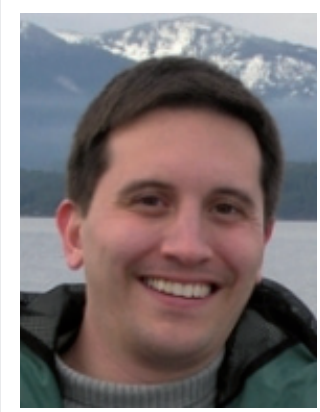

Eric T Lagally

Author for correspondence: Michael Smith Laboratories,

The University of British Columbia,

Vancouver, BC Canada V6T IZ4

Tel.: + I 6048274274

Fax: + 6048222114

E-mail: lagally@msl.ubc.ca

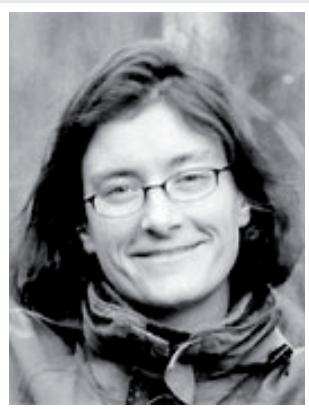

Joanne A Fox

Michael Smith Laboratories,

The University of British Columbia,

Vancouver, BC Canada V6T IZ4 
has added valuable ideas to the research programs of more than one faculty member. iGEM is now strongly supported on campus by both the Faculties of Applied Science and Science, and has helped us engage with local industry. We are also are actively reaching out to other disciplines, including the humanities, for help in learning about the human-practices aspect of this new field.

\section{"We have found numerous advantages to the integration of science outreach and research ... special effort must be made to create an environment in which these synergies can flourish."}

A second project integrating the research of the MSL faculty with the science outreach arm can be found in the recently published Jell-O microfluidics method [1]. This idea was first raised by Joanne Fox and David Ng as part of a carpool with Eric Lagally. We wanted a way to present the ideas and concepts of microfluidics that would be accessible to younger students and the general public. This introduced several design constraints, notably that such devices would have to be simple, inexpensive and fast to fabricate, but would have to be capable of demonstrating microfluidic concepts in action. It is worth noting that these design constraints are shared with another growing application space for microfluidics, that of global health. Along with two high school students working in the MSL on a semester-long internship, the Lagally group began investigating the use of Jell-O, the popular gelatin-based dessert, to fabricate microfluidics. What emerged was a simple molding procedure using coffee stirrers and tape, followed by pouring a concentrated Jell-O mixture onto the molds and overnight curing in a refrigerator. The resulting chips demonstrate concepts including laminar flow, $\mathrm{pH}$ sensing, diffusion and parallel analysis.

Like the iGEM project, there have been benefits to both the faculty and students from Jell-O microfluidics. First, the science outreach and research arms of MSL are now linked more closely than they were before, enabling a new applicationfocused side to the research in the Lagally laborataory. Second, links to other researchers across the world have resulted from our publication of this very simple method. Researchers in Australia, the USA, Canada and Europe have contacted us, widening our professional networks and promoting exploration of new ideas related to our basic research program. Third, the students leading the Jell-O workshops as well as those participating and mentoring the iGEM team have acquired a set of valuable 'soft skills', including improved written and oral communication, proposal writing, fundraising and teamwork.

Finally, with these outreach activities we have engaged students in science who would not otherwise have considered it as a career. The Jell-O lab-on-a-chip activity was first demonstrated to more than 100 high school students who visited the MSL for our annual science education conference. Survey results following these conferences indicated new interest in science from a wide crosssection of the attendees. In another example, two students from a local high school won an award at the regional science fair using the Jell-O microfluidic technique, the female member of the team remarked later that she had not considered science as a career track previously, but was doing so now.

In summary, we have found numerous advantages to the integration of science outreach and research activities, but special effort must be made to create an environment in which these synergies can flourish. Our hope is that the benefits of this combination of approaches can continue to be realized for scientists and students in academic and industrial settings.

\section{Financial \& competing interests disclosure The authors have no relevant affliations or financial involve- ment with any organization or entity with a financial interest in or financial conflict with the subject matter or materials discussed in the manuscript. This includes employment, con- sultancies, honoraria, stock ownership or options, expert testimony, grants or patents received or pending, or royalties. \\ No writing assistance was utilized in the production of this manuscript.}

\section{Bibliography}

1 Yang CWT, Ouellet E, Lagally ET. Using inexpensive Jell- ${ }^{\circledR}$ chips for hands-on microfluidics education. Anal Chem. 82(13), 5408-5414 (2010).

2 Felder RM. The link between research and teaching: 1. Does it exist? Chem. Eng. Educ. 44(2), 109-110 (2010).
3 Prince MJ, Felder RM, Brent R. Does faculty research improve undergraduate teaching? An analysis of existing and potential synergies. J. Eng. Educ. 96(4), 283 (2007).

4 Levskaya A, Chevalier AA, Tabor JJ et al. Synthetic biology: engineering Escherichia coli to see light. Nature 438 (7067), 441-442 (2005).
5 CigliC M, Fekonja O, KovaC J et al. Engineered human cells: say no to sepsis. IET Synth. Biol. 1(1-2), 13-16 (2007).

\section{- Website}

101 iGEM 2010. http://2010.igem.org 